\title{
Descripción de factores de riesgo para mortalidad en adultos con enfermedad renal crónica en estadio 3 - 5
}

\section{A description of risk factors for mortality in adults with end-stage chronic kidney disease}

\section{Correspondencia}

Carlos Hernán Calderón Franco cacalderon190@gmail.com

\section{Recibido: 22/05/2020}

Arbitrado por pares

Aprobado: 25/06/2020

Citar como: Alvis-Peña D, CalderónFranco C. Descripción de factores de riesgo para mortalidad en adultos con enfermedad renal crónica. Acta Med Peru. 2020;37(2):163-8. doi: $h$ ttps://doi.org/10.35663/ amp.2020.372.980
Diego Alvis-Peña 1,2,a, Carlos Calderón-Franco ${ }^{1,3, a}$.

Universidad Sur Colombiana. Neiva, Colombia.

Grupo de Investigación Infecto Control. Hospital Universitario Hernando Moncaleano Perdomo. Neiva, Colombia.

Clínica Medilaser. Florencia, Colombia.

Médico-cirujano, magíster en Epidemiología

\section{RESUMEN}

Objetivo: describir las características demográficas y clínicas de los pacientes con enfermedad renal crónica (ERC) en estadio 3-5 y la asociación de variables con mortalidad. Materiales y Métodos: estudio observacional, descriptivo y transversal, con enfoque analítico; Realizado en Florencia Caquetá, en una IPS privada en el servicio de nefrología, se analizaron las variables sociodemográficas, clínicas, a través de análisis univariado y bivariado, aplicando prueba de asociación con mortalidad mediante chi cuadrado y regresión logística simple para calcular razones de prevalencia (PR). Resultados: se evaluaron a 213 pacientes, con un promedio de edad de $60,0 \pm 1,4$ años; y un $48,3 \%$ de mujeres, con una mortalidad registrada de $29,5 \%$. Se hallaron como factores asociados a mortalidad: LDL < $70 \mathrm{mg} / \mathrm{dL}$ (PR 0,45; IC95\%: 0,24-0,84), y Albumina $<3,5 \mathrm{mg} / \mathrm{dL}$ (PR 12,65; IC95\%: 5,85-27,3). Conclusión: la hipoalbuminemia y los niveles bajos de LDL se presentan como factores asociados a la mortalidad en pacientes con ERC en estado 3-5 de Florencia-Caquetá. En el caso de la hipoalbuminemia, ésta se presenta como un factor de riesgo, mientras que los valores de LDL menores a $70 \mathrm{mg} / \mathrm{dL}$, son un factor protector.

Palabras clave: Insuficiencia renal crónica; Fallo renal crónico; Terapia de reemplazo renal; Diálisis renal; Diálisis peritoneal (fuente: DeCS BIREME). 


\section{ABSTRACT}

Objective: To describe both clinical and demographic characteristics of patients with end-stage kidney disease (ESKD) and the association of such variables with mortality. Materials and Methods: This is an observational, descriptive, and cross-sectional study with an analytical approach, which was performed in the nephrology service of a private HMO in Florencia-Caqueta. We studied socio-demographic and clinical variables using univariate and bivariate analyses, applying an association test with mortality using the Chi-square test and simple logistic regression, aiming to calculate prevalence rates (PR). Results: Twohundred and thirteen patients were assessed, their average age was $60.0 \pm 1.4$ years; $48.3 \%$ were female, and the recorded mortality rate was $29.3 \%$. Factors associated with mortality were as follows: LDL-C $<70 \mathrm{mg} / \mathrm{dL}$ (PR: $0.45,95 \% \mathrm{Cl}: 0.24-0.84$ ) and serum albumin <3.5 g/dL (PR: 12.65; 95\% Cl: 5.85-27.3). Conclusion: Hypoalbuminemia and low LDL-C levels are associated with mortality in patients with ESKD in Florencia-Caqueta. Hypoalbuminemia is considered as a risk factor, while LDL levels $<70 \mathrm{mg} / \mathrm{dL}$ are considered as a protective factor.

Keywords: Renal insufficiency, chronic; Kidney failure, chronic; Renal replacement therapy; Renal dialysis; Peritoneal dialysis (source: MeSH NLM).

\section{INTRODUCCIÓN}

La enfermedad renal crónica (ERC) es una enfermedad de interés en salud pública, convirtiéndose en los últimos años en una de las patologías no transmisibles más importantes afectando a millones de personas a nivel mundial, de los cuales el $80 \%$ provienen de países en vías de desarrollo ${ }^{[1]}$. Adicionalmente, se ha convertido en una enfermedad con un gran impacto económico, por los aumentos considerables que implica en los gastos del sistema de salud ${ }^{[2]}$. Es así que el Fondo Colombiano de Enfermedades de Alto Costo informa que más de 2000 pacientes requieren terapia dialítica, con una prevalencia estimada de 434 pacientes en diálisis por millón de habitantes ${ }^{[3]}$.

En Colombia, para el año 2014, se evidenció que cerca de 3 055568 de personas presentan esta enfermedad en diferentes estadios, usualmente asociados a hipertensión arterial (HTA) y Diabetes tipo 2 (DM2), perjudicando los ingresos económicos y estilos de vida de las personas afectadas ${ }^{[4]}$. Así mismo, el aumento de la prevalencia asociada a las complicaciones de la ERC, a nivel mundial, hace que sea muy difícil diseñar intervenciones adecuadas para disminuir la carga de enfermedad en los sistemas de salud ${ }^{[5,6]}$.

Por otro lado, la ERC en estadio 5 o terminal, requiere una terapia de reemplazo renal (TRR) como lo es la terapia dialítica o el trasplante renal; lo que implica un incremento significativo en los costos de atención y una afectación negativa en la calidad de vida en estos pacientes ${ }^{[7,8]}$. En los últimos años se ha observado un aumento proporcional de la ERC en los departamentos del Huila y Caquetá, lo que crea la necesidad de caracterizar a los individuos en el sur del país con ERC en estadio 5, programados a diálisis, para generar conocimiento que ayude a disminuir los costos asociados y la carga de enfermedad en el sistema de salud ${ }^{[3,9]}$.

Por lo cual, el objetivo de este estudio es describir las características demográficas y clínicas de los pacientes con Enfermedad Renal Crónica (ERC) en estadio 3-5, que ingresan a diálisis, y la asociación de estas variables con mortalidad, en una unidad de la ciudad de Florencia entre el año 2013 al 2018.

\section{MATERIALES Y MÉTODOS}

\section{Diseño y tipo de estudio}

Estudio observacional de corte transversal.

\section{Población y muestra}

El estudio se realizó con los pacientes que ingresaron a hemodiálisis en una unidad de nefrología, de la ciudad de Florencia. La población de estudio fue incluida en un periodo comprendido entre el 01 de enero del 2013 al 30 de junio del 2018.

Se incluyeron pacientes mayores de 18 años con diagnóstico de ERC estadio 3-5 (ERC 3-5), con antecedentes de HTA y/o DM2 que requirieron y/o se encontraban en TRR. Se excluyeron las historias clínicas de los pacientes con diagnóstico de insuficiencia renal aguda o con trasplante renal. Así mismo se excluyó a los registros en los que no se encontró el consentimiento informado para el manejo médico, asistieron a otro centro de salud para terapia dialítica o presentaron información incompleta.

La unidad de información fueron las historias clínicas, y no se calculó un tamaño muestral debido a que se incluyeron todos los pacientes que cumplieron con los criterios de selección establecidos $(n=213)$.

\section{Variables}

Se estudiaron variables sociodemográficas como edad, sexo, régimen de Entidad Promotora de Salud (EPS), grupo étnico y lugar de procedencia. Por otro lado, también se incluyeron variables clínicas, como medidas antropométricas, comorbilidades (HTA, DM2) y sus esquemas terapéuticos; así 
como, variables laboratoriales como creatinina, fosforo sérico, colesterol (total, LDL y HDL) y hemoglobina glicosilada (HbA1c). Adicionalmente, se evaluó diagnóstico de anemia en función a los valores de hemoglobina (anemia leve 9-11 g/dL, moderada 7-9 g/ $\mathrm{dL}$, severa $<7 \mathrm{~g} / \mathrm{dL}$ ); y estado nutricional en función a los niveles de albumina (clasificada en: normal $>3,5 \mathrm{~g} / \mathrm{dL}$, desnutrición leve 3,0-3,5 g/dL, moderada 2,5-2,9 g/dL, severa $<2,5 \mathrm{~g} / \mathrm{dL}$ ). Así mismo, se calcularon indicadores clínicos específicos de la enfermedad como la tasa de filtración glomerular (TFG) o la eficiencia de la diálisis mediante el Kt/V.

La variable dependiente del estudio fue el estado vital al egreso (de alta o fallecido).

\section{Análisis estadístico}

La información fue recolectada mediante un instrumento previamente validado por una prueba piloto, y los datos se analizaron con el paquete estadístico STATA, v 15.0 (StataCorp. 2017. College Station, TX: StataCorp LLC ${ }^{\circledR}$ ). Se realizó un análisis descriptivo con frecuencias y proporciones para las variables cualitativas y medidas de tendencia central y de dispersión para las variables cuantitativas.

Se determinó la comparación de variables cuantitativas mediante la prueba $t$ de Student para grupos independientes; y para las variables cualitativas se realizó la prueba Chi cuadrado. Así mismo, se hizo un modelo de regresión logística binaria para evaluar la asociación de variables sociodemográficas y clínicas, con el estado vital al egreso, obteniéndose razones de prevalencia (PR) con intervalos de confianza al 95\% (IC 95\%). Se determinó como nivel de significancia estadística un valor $p<0,05$.

\section{Aspectos éticos}

El protocolo recibió el aval del comité de ética del centro de referencia implicada en la investigación, en la categoría de investigación sin riesgo. Se respetaron los principios éticos establecidos para la declaración de Helsinki; por lo que no se recolecto información relacionada a datos personales de los pacientes ${ }^{[10,11]}$.

\section{RESULTADOS}

Al evaluar las características demográficas de los pacientes incluidos, la edad promedio fue de $60,0 \pm 1,4$ años, y el $48,3 \%$ perteneció al sexo femenino. Se observó que la aseguradora que tiene más pacientes es Asmet Salud $(67,4 \%)$, siendo el régimen subsidiado el más frecuente (69,9\%). El municipio de Florencia fue el que más pacientes aporto al estudio (99,2\%) (Tabla 1).

La comorbilidad más frecuente fue la presencia de hipertensión arterial (100\%), seguido de diabetes tipo 2 (62,9\%). La mayoría de pacientes presentaron una presión arterial sistólica (PAS) $>140 \mathrm{~mm} \mathrm{Hg}(54,4 \%)$ y diastólica (PAD) < $80 \mathrm{mmHg}(54,4 \%)$. El $3,3 \%$ presentó una tasa de filtración glomerular (TFG) de 8,2 \pm
Tabla 1. Características sociodemográficas de los pacientes con enfermedad renal crónica de la unidad de hemodiálisis.

\begin{tabular}{lc}
\hline \multicolumn{1}{c}{ Características } & $\mathbf{n}(\%)$ \\
\hline Edad $^{*}$ & $61 \pm 13,5$ \\
Sexo & \\
Femenino & $103(48,4)$ \\
Masculino & $110(51,6)$ \\
Régimen de EPS & \\
Subsidiado & $149(70,3)$ \\
Contributivo & $59(27,7)$ \\
Especial & $5(2,3)$ \\
Grupo Étnico & \\
Ninguno & $212(99,5)$ \\
Afroamericano & $1(0,5)$ \\
Municipio & \\
Florencia & $211(99,1)$ \\
Currillo & $1(0,5)$ \\
Puerto Rico & $1(0,5)$ \\
\hline
\end{tabular}

* media \pm desviación estándar

EPS: entidad prestadora de salud

$3,3 \mathrm{~mL} / \mathrm{min} / 1,73 \mathrm{~m}^{2}$; mientras que, el modelo de diálisis primario más frecuente fue por urgencias $(74,1 \%)$. Los pacientes que recibieron tratamiento antihipertensivo con inhibidores de la enzima convertidora de angiotensina (IECA) fueron del 14,5\%; mientras que los que recibieron tratamiento con antagonistas del receptor de angiotensina II (ARA-2) fueron el 15,9\%. (Tabla 2).

Los pacientes que tenían una $\mathrm{HbA} 1 \mathrm{C}>7 \%$, fueron del $58,0 \%$. Se observó un promedio de colesterol total de $170,0 \mathrm{mg} / \mathrm{mL}$ (rango $42,8-351,0 \mathrm{mg} / \mathrm{dL}$ ), de igual forma el colesterol HDL tuvo una media de $40,3 \mathrm{mg} / \mathrm{mL}$ y el colesterol LDL, de $93,9 \mathrm{mg} / \mathrm{mL}$ (Tabla 2).

Se observó que las personas evaluadas, en general, tuvieron niveles normales de albumina $(80,2 \%)$; sin embargo, es importante resaltar que el 6,1\% presentó desnutrición grave. La mayoría de pacientes tenían anemia leve (47,4\%), y 57,2\% presentaron fosforo normal. Se observó que la dosis Kt/V promedio fue de 1,69 (rango 0,3-5,0). En total fallecieron el 29,5\% de los pacientes, con un promedio de edad de fallecimiento 65,7 años.

De acuerdo con los resultados obtenidos, se visualiza que la presencia de DM2 y la TFG $<30 \mathrm{~mL} / \mathrm{min}$ fueron las variables más frecuentes en los pacientes que fallecieron; mientras que, en menor medida se observó la presencia de un IMC mayor a $25 \mathrm{~kg} / \mathrm{m}^{2}$. Los niveles de presión arterial (PAS y PAD) y la TFG $<30 \mathrm{~mL} / \mathrm{min}$ no presentaron asociación estadística significativa para mortalidad. Para los pacientes que recibieron tratamiento antihipertensivo con IECA y ARA II, tampoco se encontró asociación estadística significativa con relación a la mortalidad. Las variables analizadas que tuvieron asociación significativa fueron niveles de albumina $<3,5 \mathrm{mg} / \mathrm{dL}$, LDL menor a $70 \mathrm{mg} / \mathrm{dL}$ (Tabla 3). Asi mismo, se evidenciaron diferencias significativas 
Tabla 2. Características clínicas de los pacientes con enfermedad renal crónica de la unidad de hemodiálisis.

\begin{tabular}{|c|c|}
\hline Características & n (\%) \\
\hline \multicolumn{2}{|l|}{ Comorbilidades } \\
\hline HTA & $213(100,0)$ \\
\hline DM2 & $134(62,9)$ \\
\hline \multicolumn{2}{|l|}{ Etiología ERC } \\
\hline HTA o DM2 & $210(98,6)$ \\
\hline Nefropatía obstructiva & $1(0,5)$ \\
\hline Autoinmune & $2(0,9)$ \\
\hline Edad Diagnóstico $\left.{ }^{*}\right)$ & $58,0 \pm 14,9$ \\
\hline Peso $(\mathrm{kg})^{(*)}$ & $62,7 \pm 14,2$ \\
\hline Talla $(m)^{(*)}$ & $1,6 \pm 0,1$ \\
\hline PAS $(\mathrm{mmHg})^{(*)}$ & $143,0 \pm 22,0$ \\
\hline$<120$ & $37(17,4)$ \\
\hline $120-129$ & $28(13,1)$ \\
\hline $130-139$ & $32(15,0)$ \\
\hline$>140$ & $116(54,5)$ \\
\hline $\operatorname{PAD}(\mathrm{mm} \mathrm{Hg})^{(*)}$ & $80,0 \pm 12,0$ \\
\hline$<80$ & $116(54,5)$ \\
\hline $80-89$ & $45(21,1)$ \\
\hline$>90$ & $52(24,4)$ \\
\hline Creatinina $(\mathrm{mg} / \mathrm{dL})^{(*)}$ & $8,2 \pm 3,38$ \\
\hline TFG inicial ${ }^{* *}$ & $9,9 \pm 5,8$ \\
\hline Colesterol (mg/dL) (*) & $170,9 \pm 49,7$ \\
\hline $\mathrm{HDL}(\mathrm{mg} / \mathrm{dL})^{(*)}$ & $40,3 \pm 16,2$ \\
\hline $\mathrm{LDL}(\mathrm{mg} / \mathrm{dL})^{(*)}$ & $93,9 \pm 38,9$ \\
\hline Clasificación HbA1c (*) & $7,8 \pm 2,4$ \\
\hline$<7 \%$ & $13(6,1)$ \\
\hline$\geq 7 \%$ & $18(8,5)$ \\
\hline Hemoglobina ${ }^{(*)}$ & $10,9 \pm 8,7$ \\
\hline Anemia severa & $26(12,2)$ \\
\hline Anemia moderada & $57(26,8)$ \\
\hline Anemia leve & $101(47,4)$ \\
\hline Normal & $29(13,6)$ \\
\hline \multicolumn{2}{|l|}{ Estado de ERC } \\
\hline $30-50 \mathrm{~mL} / \mathrm{min}$ & $2(0,9)$ \\
\hline $15-30 \mathrm{~mL} / \mathrm{min}$ & $23(10,8)$ \\
\hline$<15 \mathrm{~mL} / \mathrm{min}$ & $188(88,3)$ \\
\hline \multicolumn{2}{|l|}{ Modelo de inicio TRR } \\
\hline Urgencias & $158(74,2)$ \\
\hline Programada & $17(8,0)$ \\
\hline No se conoce & $37(17,4)$ \\
\hline Otra EPS & $1(0,5)$ \\
\hline Dosis Kt/V Total ${ }^{(*)}$ & $1,7 \pm 1,0$ \\
\hline Albúmina $(\mathrm{mg} / \mathrm{dL})^{(*)}$ & $10,5 \pm 24,1$ \\
\hline Desnutrición grave & $13(6,1)$ \\
\hline Desnutrición moderada & $7(3,3)$ \\
\hline Desnutrición leve & $22(10,3)$ \\
\hline Normal & $171(80,3)$ \\
\hline Fósforo $(\mathrm{mg} / \mathrm{dL})^{(*)}$ & $4,0 \pm 1,5$ \\
\hline Hipofosfatemia & $28(13,1)$ \\
\hline Hiperfosfatemia & $63(29,6)$ \\
\hline Normal & $122(57,3)$ \\
\hline Mortalidad & $63(29,5)$ \\
\hline Edad de fallecimiento & $65,7 \pm 10,0$ \\
\hline
\end{tabular}

(*) Media \pm desviación estándar

TFG calculada por CKD-EPI en $\mathrm{mL} / \mathrm{min} / 1,73 \mathrm{~m} 2$

TRC: tasa de reemplazo renal; ERC: enfermedad renal crónica; HTA: hipertensión arterial; DM2: diabetes tipo 2; TRR: terapia de reemplazo renal; TFG: tasa de filtración glomerular; PAS: presión arterial sistólica; PAD: presión arterial diastolica. HDL: colesterol de alta densidad; LDL: colesterol de baja densidad; HbA1c: hemoglobina glicosilada. $(p<0,001)$ entre los promedios de las edades de pacientes que fallecieron $(66,0 \pm 1,4$ años) y los que permanecieron vivos (58,0 $\pm 1,1$ años).

\section{DISCUSIÓN}

En el presente estudio se realizó una descripción a 213 pacientes, que ingresaron al programa de hemodiálisis crónica durante un periodo de 5 años; encontrando una edad promedio de fallecimiento fue de 66 años, con un rango entre 29 a 85 años. Estos hallazgos son similares a lo evidenciado en el estudio de Soucie et al., (1996) y Collings et al., (2015), realizados en registros norteamericanos ${ }^{[12,13]}$.

La mortalidad encontrada en nuestro estudio fue de $29,5 \%$, mayor que la reportada en los otros estudios de otras instituciones que oscila entre 6 y $12 \%{ }^{[12,13]}$. La edad promedio de los pacientes (60 $\pm 13,5$ años), concuerda con los hallazgos reportados en otros estudios como los de Ortega et al., (2006) y Metcalfe et al., (2000), que describieron un promedio de edad entre los 64 y 71 años ${ }^{[14,15]}$. De igual manera la enfermedad es más frecuente en varones $(51,7 \%)$ que se encuentran en edades laboralmente activas, lo cual supone una rápida evolución a enfermedad renal terminal lo cual influye en la calidad de vida de los pacientes y repercute en el orden económico ${ }^{[16]}$.

La existencia de múltiples comorbilidades como la HTA (100\%) o la DM2 (62,9\%) es frecuente en los pacientes que ingresan a terapia de reemplazo renal y es más evidente en los que llegan de forma tardía al tratamiento ${ }^{[17]}$. A su vez, el riesgo de muerte es mayor en la medida en que exista mayor comorbilidad y descompensación de las patologías, mostrando un comportamiento similar a lo descrito por Ortega et al. ${ }^{[14]}$.

La ERC se asocia con enfermedades crónicas ya que un $25-40 \%$ de los pacientes diabéticos presentarán algún grado de nefropatía; y un $38,1 \%$ de las personas que tienen hipertensión arterial, también tienen ERC ${ }^{[18]}$. Además, el $30,7 \%$ de los hipertensos del país no ha sido estudiado para ERC ${ }^{[19]}$. Este estudio presenta datos similares, siendo coherente con la identificación de DM2 y HTA como principales antecedentes de los pacientes con ERC-5, con terapia de reemplazo renal. Lo anterior manifiesta la necesidad de realizar una evaluación temprana de los pacientes para realizar acciones con el fin de evitar la presentación de la enfermedad y que esta llegue a estadios más avanzados con daños irreversibles.

En nuestros resultados se evidenció que todos los pacientes con ERC terminal recibieron terapia de reemplazo renal, lo cual puede haber sido por la concomitancia y asociación de patologías (HTA-DM2), que ayudan a progresar rápidamente a estados avanzados de la ERC, y aumenta el requerimiento de TRR como parte del manejo y tratamiento integral. Sin embargo, en un estudio realizado con pacientes con ERC en Colombia concluyó que la mayoría de los pacientes con ERC-5 están en hemodiálisis $(67,6 \%)$. Esta situación contrasta con cifras a nivel mundial, para 
Tabla 3. Características clínicas de los pacientes con enfermedad renal crónica de la unidad de hemodiálisis ${ }^{*}$ ).

\begin{tabular}{|c|c|c|c|c|c|}
\hline Características & $\begin{array}{c}\text { Muertos ( } n=63) \\
n(\%)\end{array}$ & $\begin{array}{c}\text { Vivos }(n=150) \\
n(\%)\end{array}$ & Valor $p$ & PR & IC 95\% \\
\hline Sexo (masculino) & $30(47)$ & $80(53)$ & 0,446 & 0,79 & $(0,44-1,43)$ \\
\hline Diagnóstico de DM2 & $44(70)$ & $60(40)$ & 0,175 & 0,64 & $(0,34-1,21)$ \\
\hline $\mathrm{IMC}>25 \mathrm{Kg} / \mathrm{cm}$ & $22(35)$ & $67(47)$ & 0,188 & 0,66 & $(0,36-1,22)$ \\
\hline $\mathrm{PAD}>90 \mathrm{mmHg}$ & $10(16)$ & $29(24)$ & 0,551 & 0,78 & $(0,35-1,73)$ \\
\hline PAS > $130 \mathrm{mmHg}$ & $38(60)$ & $106(71)$ & 0,141 & 0,63 & $(0,34-1,16)$ \\
\hline $\mathrm{TFG}<30 \mathrm{ml} / \mathrm{min} / \mathrm{kg}$ & $62(98)$ & $148(99)$ & 0,886 & 0,83 & $(0,07-9,40)$ \\
\hline $\mathrm{LDL}<70 \mathrm{mg} / \mathrm{dL}$ & $36(57)$ & $112(75)$ & 0,011 & 0,45 & $(0,24-0,84)$ \\
\hline Tratamiento IECA & $7(11)$ & $24(16)$ & 0,356 & 0,65 & $(0,26-1,61)$ \\
\hline Tratamiento ARA II & $8(12)$ & $26(17)$ & 0,399 & 0,69 & $(0,29-1,64)$ \\
\hline Albumina $<3,5 \mathrm{mg} / \mathrm{dL}$ & $33(52)$ & $12(8)$ & $<0,001$ & 12,65 & $(5,85-27,3)$ \\
\hline
\end{tabular}

$(*)$ Las variables presentadas en la tabla corresponden a información emparejada, en algunas variables no fue posible recolectar la información para las 213 observaciones. Solo se colocaron las categorías de interés.

DM2: diabetes tipo 2; IMC: índice de masa corporal; PAD: presión arterial diastólica; PAS: presión arterial sistólica; TFG: tasa de filtración glomerular; LDL: colesterol de baja densidad; IECA: inhibidores de la enzima convertidora de angiotensina; ARA II: antagonistas de los receptores de la angiotensina II.

el año 2010, donde se estima que 2618 millones de personas en el mundo recibieron TRR, de las cuales 2050 millones (78\%) recibieron diálisis y el resto, trasplante ${ }^{[19,20]}$.

Los pacientes con insuficiencia renal son un grupo de alto riesgo nutricional, por esta razón la albúmina sérica es un marcador nutricional utilizado para identificar la desnutrición en pacientes con ERC. Varios estudios han reflejado que niveles de albúmina sérica inferiores a $3,5 \mathrm{~g} / \mathrm{dL}$ son un importante predictor de la tasa de mortalidad y hospitalización en pacientes crónicos en hemodiálisis ${ }^{[20,21]}$. En este estudio, se observan datos similares, ya que, del total de los registros estudiados, el $50 \%$ de los pacientes que fallecieron tenían niveles de albumina $<3,5 \mathrm{mg} / \mathrm{dL}$; mientras que estos valores solo se encontraron en el $8 \%$ de los pacientes que sobrevivieron. Adicionalmente, estas cifras de albumina sérica estuvieron estadísticamente asociadas con la mortalidad, concluyéndose que en pacientes con ERC 3-5, esta condición está relacionada con un riesgo 11,6 veces mayor de morir.

Otros factores asociados con mortalidad en diálisis descritos en la literatura son: la raza ${ }^{[19]}$, la dosis Kt/ $\mathrm{V}{ }^{[22,23]}$, los factores psicosociales ${ }^{[24]}$, hipo o hipercolesterolemia, valores de hemoglobina inferiores a $10 \mathrm{~g} / \mathrm{dL}$, parathormona baja o elevada, un nivel bajo de homocisteína y de vitamina B6 [25,26]. Muchos de estos factores no fueron caracterizados en este estudio, por lo que su relación con la mortalidad es aún desconocida. Se sugiere que futuros estudios consideren a estas variables o de lo contrario controlen estos factores al momento de analizar los datos recolectados.

Nuestro estudio presentó algunas limitaciones como la evaluación retrospectiva que no permite hacer controles adecuados, ni permite la determinación de una relación causal (causa-efecto o efecto-causa) por lo cual, podremos aproximar, pero no distinguir con exactitud los factores de riesgo y los factores pronósticos en esta población. Por otro lado, es probable que se encuentre sesgo de información, secundario al diligenciamiento incompleto de las historias clínicas o por falta de datos confiables. Así mismo, se debe considerar que se evaluó a un solo centro, por lo que las conclusiones de esta investigación son solo aplicables a este ámbito. Sin embargo, este centro de referencia es la única institución de mayor complejidad con unidad de diálisis en la región; y a nivel regional y local, no se han encontrado estudios similares realizados en población con ERC 3-5 en TRR. Por ende, el estudio aporta información valiosa sobre el comportamiento local de la enfermedad y sirve como un marco referencial para generar nuevas investigaciones.

En conclusión, se encontró una frecuencia de mortalidad de $29,5 \%$, con niveles de albumina sérica inferiores a 3,5 g/dL, colesterol LDL menor a 70 y edad mayor a 60 años asociados con la mortalidad de pacientes con IRC-5.

Los pacientes que iniciaron hemodiálisis fueron en su mayoría hombres, de edad avanzada, del régimen subsidiado y área urbana de Florencia, con múltiples comorbilidades (HTA, DM2), con reporte de paraclínicos que evidenciaban, niveles altos de $\mathrm{HbA1c}$, anemia y niveles altos de LDL.

Contribuciones de autoría: todos los autores declaran haber participado en el diseño y concepción del estudio, la recolección y análisis de datos; así como en la elaboración y aprobación del manuscrito final para publicación. Así mismo, todos los autores se comprometen a responder sobre todos los aspectos del artículo de cara a asegurar que las cuestiones relacionadas con la exactitud o integridad de cualquier parte del trabajo están adecuadamente investigadas y resueltas.

Potenciales conflictos de interés: los autores declaran no tener conflictos de interés

Fuente de financiamiento: autofinanciado 
ORCID:

Diego Alvis-Peña: https://orcid.org/0000-0002-6885-0816

Carlos Calderón-Franco: https://orcid.org/0000-0002-9823-8409

\section{REFERENCIAS BIBLIOGRÁFICAS}

1. Stanifer JW, Muiru A, Jafar TH, Patel UD. Chronic kidney disease in low- and middle-income countries. Nephrol Dial Transplant. 2016;31(6):868-74. doi: 10.1093/ndt/gfv466.

2. Sistema General de Seguridad Social en Salud. Guía Práctica Clínica para el diagnóstico y tratamiento de la Enfermedad Renal Crónica (adopción) [Internet]. Bogotá: Ministerio de Salud y Protección Social; 2016 [citado 20 junio 2020]. Disponible en: http://gpc. minsalud.gov.co/gpc_sites/Repositorio/Otros_conv/GPC_e_renal/ GPC_enfermedad_renal_adopcion.pdf.

3. Fondo Colombiano de Enfermedades de Alto Costo Cuenta de Alto Costo. Situación de la Enfermedad Renal Crónica, la Hipertensión Arterial y la Diabetes Mellitus en Colombia 2017 [Internet]. Bogotá: Cuentas de Alto Costo; 2017 [citado 20 junio 2020]. Disponible en: https://cuentadealtocosto. $\mathrm{org} / \mathrm{site} /$ publicaciones/situacion-de-la-enfermedad-renalcronica-la-hipertension-arterial-y-la-diabetes-mellitus-encolombia-2017/?1593490948473.

4. Mills KT, Xu Y, Zhang W, Bundy JD, Chen C-S, Kelly TN, et al. A systematic analysis of world-wide population-based data on the global burden of chronic kidney disease in 2010. Kidney Int 2015;88(5):950-7. doi: 10.1038/ki.2015.230

5. Hill NR, Fatoba ST, Oke JL, Hirst JA, O'Callaghan CA, Lasserson DS, et al. Global Prevalence of Chronic Kidney Disease - A Systematic Review and Meta-Analysis. PLoS ONE 2016;11(7):e0158765. doi: 10.1371/journal.pone.0158765

6. Academy of Nutrition and Dietetics. CKD: Major Recommendations (2010) [Internet]. En: 2010 Chronic Kidney Disease (CKD) EvidenceBased Nutrition Practice Guideline. Chicago: Academy of Nutrition and Dietetics (Academy); 2010 [citado 20 junio 2020]. p. 56. Disponible en: https://www.andeal.org/vault/pqnew95.pdf.

7. Espinosa-Cuevas M de los Á. Enfermedad renal. Gac Med Mex. 2016;152(S1):90-6.

8. Gómez Carracedo A, Arias Muñana E, Jiménez Rojas C. Insuficiencia Renal Crónica [Internet]. En: Tratado de Geriatria para residentes. Madrid: Sociedad Española de Geriatria y Gerontología; 2006 [citado 20 junio 2020]. p. 637-46. Disponible en: https://www.segg. es/download.asp?file=/tratadogeriatria/PDF/S35-05\%2062_III.pdf.

9. Lopera Medina MM. La enfermedad renal crónica en Colombia: necesidades en salud y respuesta del Sistema General de Seguridad Social en Salud. Gerencia y Políticas de Salud 2016;15(30):212-33.

10. World Medical Association. World medical association declaration of helsinki: Ethical principles for medical research involving human subjects. JAMA 2013;310(20):2191-4. doi: 10.1001/ jama.2013.281053.

11. Ministerio de Salud (Colombia). Resolución Número 8430 DE 1993. Normas científicas, técnicas y administrativas para la investigación en salud [Internet]. 1993 [citado 20 junio 2020]. Disponible en: https://www.minsalud.gov.co/sites/rid/Lists/BibliotecaDigital/ RIDE/DE/DIJ/RESOLUCION-8430-DE-1993.PDF.
12. Soucie JM, McClellan WM. Early death in dialysis patients: risk factors and impact on incidence and mortality rates. J Am Soc Nephrol. 1996;7(10):2169-75.

13. Collins AJ, Foley RN, Gilbertson DT, Chen S-C. United States Renal Data System public health surveillance of chronic kidney disease and end-stage renal disease. Kidney Int Suppl (2011). 2015;5(1):2-7. doi: 10.1038/kisup.2015.2.

14. Ortega M, Martínez J, Gamarra G. Mortality in patients with chronic renal failure during the first 90 days of hemodialysis therapy. Acta Med Colomb. 2006;31(1):13-9.

15. Metcalfe W, Khan IH, Prescott GJ, Simpson K, MacLeod AM. Can we improve early mortality in patients receiving renal replacement therapy? Kidney Int. 2000;57(6):2539-45. doi: 10.1046/j.15231755.2000.00113.x

16. Carrero JJ, Hecking M, Chesnaye NC, Jager KJ. Sex and gender disparities in the epidemiology and outcomes of chronic kidney disease. Nat Rev Nephrol. 2018;14(3):151-64. doi: 10.1038/ nrneph.2017.181

17. Lea JP, Nicholas SB. Diabetes mellitus and hypertension: key risk factors for kidney disease. J Natl Med Assoc. 2002;94(8 Suppl):7S-15S.

18. Thawornchaisit $P$, de Looze F, Reid CM, Seubsman S, Tran TT, Sleigh A. Health-Risk Factors and the Prevalence of Chronic Kidney Disease: Cross-Sectional Findings from a National Cohort of 87143 Thai Open University Students. Glob J Health Sci. 2015;7(5):59-72. doi: 10.5539/gjhs.v7n5p59

19. Acuña L, Sánchez P, Soler LA, Alvis LF. Enfermedad renal en Colombia: prioridad para la gestión de riesgo. Rev Panam Salud Publica. 2016;40:16-22.

20. Gómez de la Torre-Del Carpio A, Bocanegra-Jesús A, Guinetti-Ortiz $\mathrm{K}$, Mayta-Tristán P, Valdivia-Vega R. Early mortality in patients with chronic kidney disease who started emergency haemodialysis in a Peruvian population: Incidence and risk factors. Nefrologia. 2018;38(4):425-32. doi: 10.1016/j.nefro.2017.11.017.

21. McClellan WM, Soucie JM, Flanders WD. Mortality in end-stage renal disease is associated with facility-to-facility differences in adequacy of hemodialysis. J Am Soc Nephrol. 1998;9(10):1940-7.

22. Port FK, Ashby VB, Dhingra RK, Roys EC, Wolfe RA. Dialysis dose and body mass index are strongly associated with survival in hemodialysis patients. J Am Soc Nephrol. 2002;13(4):1061-6.

23. Frankenfield DL, Rocco MV, Frederick PR, Pugh J, McClellan WM, Owen WF. Racial/ethnic analysis of selected intermediate outcomes for hemodialysis patients: results from the 1997 ESRD Core Indicators Project. Am J Kidney Dis. 1999;34(4):721-30. doi: 10.1016/s0272-6386(99)70399-9

24. Norris KC, Williams SF, Rhee CM, Nicholas SB, Kovesdy CP, KalantarZadeh K, et al. Hemodialysis Disparities in African Americans: The Deeply Integrated Concept of Race in the Social Fabric of our Society. Semin Dial. 2017;30(3):213-23. doi: 10.1111/sdi.12589

25. Iseki K, Yamazato M, Tozawa M, Takishita S. Hypocholesterolemia is a significant predictor of death in a cohort of chronic hemodialysis patients. Kidney Inter. 2002;61(5):1887-93. doi: 10.1046/j.15231755.2002.00324.x.

26. Nishizawa $Y$, Shoji T, Ishimura E, Inaba M, Morii H. Paradox of risk factors for cardiovascular mortality in uremia: is a higher cholesterol level better for atherosclerosis in uremia? Am J Kidney Dis. 2001;38(4 Suppl 1):S4-7. doi: 10.1053/ajkd.2001.27380. 